Le virus Zika

\title{
L'émergence d'une menace
}

\author{
Sara Salinas ${ }^{1}$, Vincent Foulongne ${ }^{1,2}$, \\ Fabien Loustalot ${ }^{1}$, Chantal Fournier-Wirth ${ }^{1,3}$, \\ Jean-Pierre Molès ${ }^{1}$, Laurence Briant ${ }^{4}$, \\ Nicolas Nagot ${ }^{1,2}$, Philippe Van de Perre ${ }^{1,2}$, \\ Yannick Simonin ${ }^{1,5}$
}

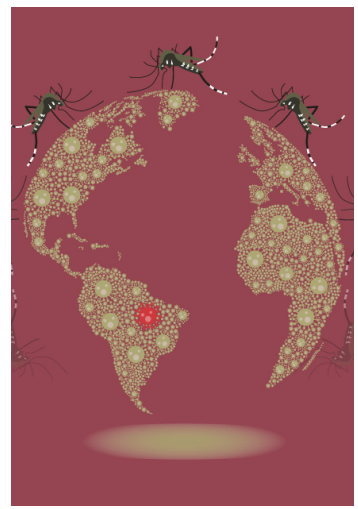

${ }^{1}$ UMR 1058, Inserm/université de Montpellier/Établissement français du sang, Pathogenesis and control of chronic infections, Inserm, 60, rue de Navacelles, 34394 Montpellier Cedex 5, France ;

${ }^{2}$ Centre hospitalier universitaire de Montpellier, département de bactériologie-virologie, Montpellier, France ;

${ }^{3}$ Établissement français du sang, parc Euromédecine,

34184 Montpellier Cedex 4,

ou encore le virus

West Nile ${ }^{2}$ (WNV)

$(\rightarrow)$ Voir la Synthèse France est avérée. L'ampleur de l'épidémie actuelle laisse apparaître l'état très primitif des connaissances concernant la physiopathologie de ce virus. Ainsi, on assiste à un effort à l'échelle mondiale afin de caractériser au plus vite l'interaction moléculaire de ce virus avec les cellules humaines mais également de développer des tests diagnostiques spécifiques et des approches vaccinales. <

\section{Le virus Zika}

Jusqu'à peu, le virus Zika (ZIKV) a beaucoup moins focalisé l'attention que d'autres arbovirus ${ }^{1}$ émergents comme le virus de la dengue (DENV) [55] $(\rightarrow)$, le virus

$(\rightarrow)$ Voir la Nouvelle de M. Flamand et P. Desprès, $m / s n^{\circ} 8$ 8-9, août-septembre 2002, page 816 $\mathrm{du}$ chikungunya $(\mathrm{CHIKV})[56](\rightarrow)$, $(\rightarrow)$ Voir la Dernière Heure de P.H. Consigny et al., $m / s n^{\circ} 4$, avril 2006, page 444

${ }^{1}$ Arbovirus signifie arthropod-borne virus: qui est porté par les arthropodes. Les arbovirus se multiplient à la fois chez les vertébrés (éventuellement l'Homme) et chez des arthropodes qui sont des insectes piqueurs (moustiques ou anophèles ou tiques). L'insecte s'infecte en aspirant le sang de ces vertébrés. Le virus se multiplie dans le tube digestif de l'insecte puis diffuse et gagne ses glandes salivaires. L'insecte transmettra le virus par sa salive au prochain vertébré qu'il va piquer.

[57] $(\rightarrow)$, cela principalement en M.C. Lanteri et al. $\mathrm{m} / \mathrm{s} \mathrm{n}^{\circ} 4$, avril 2011, page 375

raison de son faible impact épidémiologique associé à des symptômes de type pseudo-grippal. À peine plus de 100 articles ont été publiés dans le domaine depuis 1952, dont la moitié en 2015, la plupart étant des analyses épidémiologiques. On connaît ainsi très peu de choses sur son tropisme, son cycle ou encore sa pathogenèse.

Le ZIKV est un arbovirus (virus se transmettant par les arthropodes) de la famille des Flaviviridae et du genre flavivirus. II est très proche phylogénétiquement du virus Spondweni ${ }^{3}$ avec lequel il compose un clade $^{4}$ restreint [1]. Au sein des flavivirus, on retrouve quelques-uns des arbovirus les plus délétères pour l'Homme comme les virus de la dengue, de l'encéphalite japonaise, de la fièvre jaune et du Nil occidental, combinant au total plusieurs millions de cas d'infections chez I'Homme chaque année. Le ZIKV est un virus enveloppé d'environ $40 \mathrm{~nm}$ de diamètre, à ARN simple brin de polarité positive d'environ $11 \mathrm{~kb}$ [2]. II tire son nom d'une forêt de l'Ouganda, située à proximité de la capitale, Kampala, où il a été identifié pour la première fois chez

\footnotetext{
${ }^{2}$ Virus du Nil occidental.
}

${ }^{3}$ Virus responsable de la fièvre Spondweni, caractérisée par de la fièvre, des frissons, des nausées, des maux de têtes, des malaises et des épistaxis. II est retrouvé en Afrique subsaharienne et en PapouasieNouvelle-Guinée.

${ }^{4}$ Clade ou groupe monophylétique (issu d'un seul ancêtre). Un clade réunit tous les organismes qui possèdent une même innovation évolutive et un ancêtre commun, exclusif, chez qui cette innovation est apparue. 


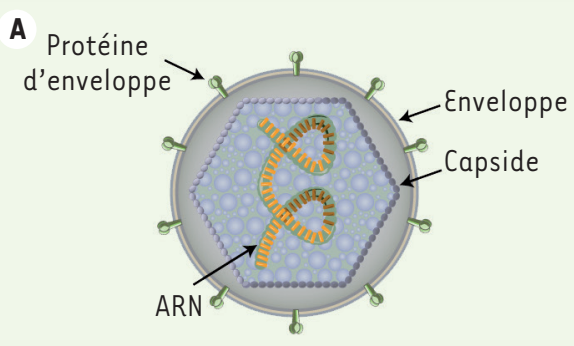

B

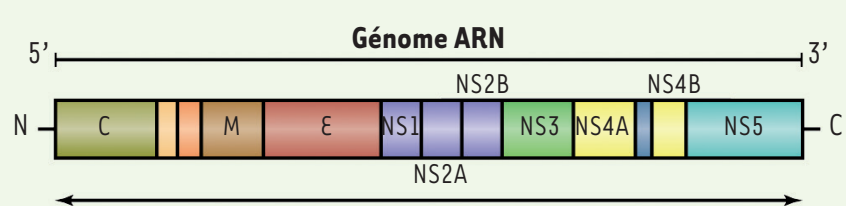

Polyprotéine

Figure 1. Structure et organisation du génome viral. A. Vue d'ensemble de la particule virale comprenant l'enveloppe et une capside de symétrie icosaédrique renfermant le génome viral. B. Représentation du génome viral. Le ZIKV possède un génome à ARN simple brin de polarité positive composé de 10 gènes flanqués de 2 régions non codantes. Un seul cadre de lecture code une polyprotéine aboutissant après clivage à la libération de 3 protéines structurales (C, prM et $\varepsilon$ ) et de 7 protéines non structurales (NS1, NS2a, NS2b, NS3, NS4a, NS4b and NS5).

un singe macaque rhésus, en 1947 [3]. II a été isolé plus tardivement chez l'Homme, en 1954 au Nigéria [4]. Des analyses phylogénétiques ont montré qu'il était composé d'un lignage africain, subdivisé en deux sous-lignages, et d'un lignage asiatique responsable de la majorité des épidémies décrites [5]. Le génome du ZIKV est composé d'un seul cadre de lecture codant une polyprotéine aboutissant, après clivage, à la libération de 3 protéines structurales ( $C$, prM et $\varepsilon$ ) et de 7 protéines non structurales (NS1, NS2a, NS2b, NS3, NS4a, NS4b and NS5) (Figure 1) [6]. La protéine C forme la capside (qui entoure I'ARN) ; la protéine prM est un précurseur de la protéine $M$ qui s'insère dans l'enveloppe du virus, le fragment pr est quant à lui excrété lors de la sortie du virus de la cellule hôte; la protéine $\varepsilon$ est la composante principale de l'enveloppe, elle est impliquée dans différents aspects du cycle viral, permettant la fixation et la fusion du virus aux cellules de l'hôte. Chez les flavivirus, les protéines non structurales s'associent aux membranes cellulaires du reticulum endoplasmique où elles forment un complexe de réplication dans lequel la protéine NS5 assure la réplication de l'ARN viral par son activité ARN-polymérase ARN dépendante. Comme pour les autres membres des flavivirus, la réplication virale s'effectue dans le cytoplasme de la cellule infectée. Cependant, des antigènes viraux peuvent se retrouver au niveau du noyau [7]. Le cycle viral du ZIKV reste très peu connu, même si l'analogie avec d'autres flavivirus semble pertinente. Une étude récente a ainsi permis de mettre en évidence différents facteurs d'attachement du ZIKV comme la lectine membranaire DC-SIGN (dendritic cell-specific intercellular adhesion molecule-3-grabbing non-integrin) et Axl et Tyro 3, des récepteurs tyrosine kinase de la famille des TAM (Tyro3, Axl, Mer) connus pour favoriser l'attachement et l'internalisation de nombreux virus dont le DENV [8]. La physiopathologie liée à l'infection ZIKV, notamment au niveau cellulaire, est largement méconnue et reste donc à explorer. II est néanmoins décrit que le ZIKV, comme d'autres flavivirus, a un fort tropisme cutané. Les kératinocytes et les fibroblastes du derme sont notamment permissifs à l'infection, ce qui permet d'envisager, pour ces populations cellulaires, un rôle majeur dans les étapes initiales de l'infection après l'inoculation cutanée du virus par le moustique [8]. Un tropisme neuronal reste quant à lui à démontrer bien que des études publiées en 1952 et 1971 aient établi dans un modèle murin qu'une injection intracérébrale de ZIKV induisait une atteinte neurologique $[9,10]$. Ainsi, dans ces expériences, Bell et al. ont détecté une réplication du virus dans les neurones et les cellules gliales associée à des inclusions intracytoplasmiques et à une dégénérescence neuronale [10]. Chez l'Homme, l'autopsie de patients infectés a révélé par RT-PCR (reverse transcriptase polymerase chain reaction) la présence d'ARN viral dans le cerveau de même que dans d'autres organes (foie, rate, rein, cœur), ce qui pose des interrogations quant aux sites de réplications du virus (voir plus loin) [11].

\section{Les vecteurs}

La transmission vectorielle du ZIKV s'effectue principalement par des moustiques du genre Aedes dont la plupart sont également vecteurs d'autres arbovirus comme DENV ou CHIKV. II existe deux cycles de transmission du virus. Un premier, le cycle sylvatique ${ }^{5}$, implique un réservoir animal, des primates non humains, et des vecteurs du genre Aedes africanus ou luteocephalus, l'Homme n'étant alors qu'un hôte «accidentel» (Figure 2). Ce cycle de transmission est responsable de la majorité des cas sporadiques. II n'y a, à ce jour, pas d'évidence de l'existence de réservoirs non primates pour le virus même si une étude a montré l'existence d'anticorps ${ }^{6}$ anti-ZIKV notamment chez de nombreux mammifères dont les rongeurs [12]. Le second cycle est de type urbain. Le virus se transmet d'Homme à Homme via des vecteurs du genre Aedes aegypti et Aedes albopictus, la survenue d'épidémies étant alors favorisée dans les zones densément peuplées (Figure 2A). La plupart des épidémies actuelles en Amérique latine se propagent par l'intermé-

\footnotetext{
${ }^{5}$ L'Homme peut être occasionnellement piqué, lors d'incursions dans les forêts et savanes limitrophes, par un moustique contaminé par le virus.

${ }^{6}$ Le portage du virus par les hôtes est suggéré par le développement d'une réponse
} immunitaire spécifique. 
A

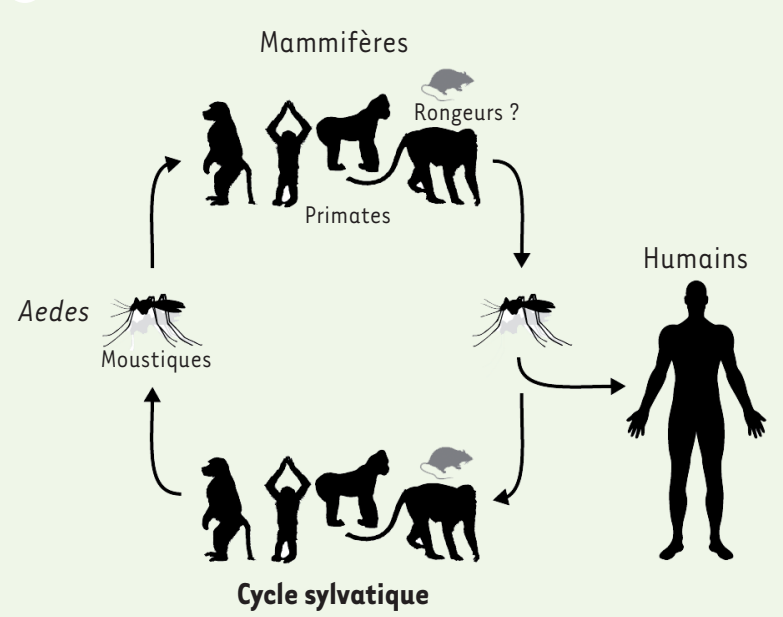

B

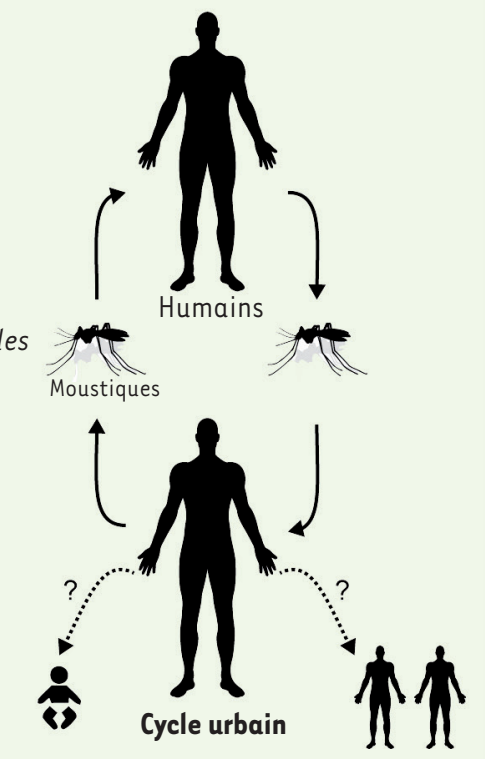

C
Organes présentant de l'ARN viral

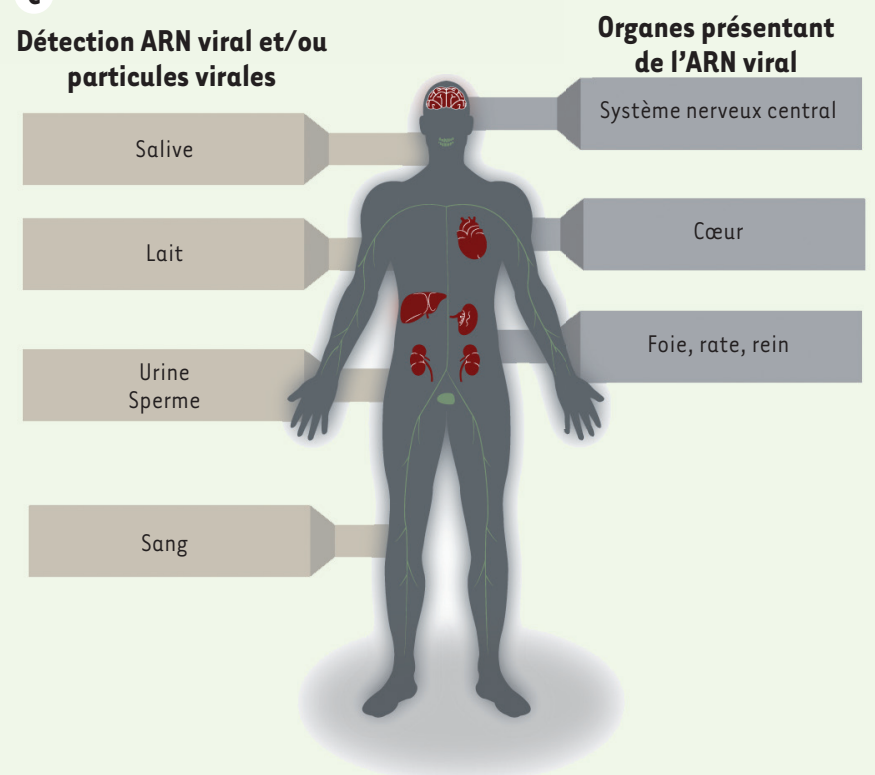

Figure 2. Cycles de transmission et organes cibles. A. Cycle sylvatique impliquant des réservoirs animaux (probablement des primates) et un vecteur du genre Aedes. L'Homme n'étant qu'un hôte accidentel de ce type de transmission. B. Cycle urbain impliquant l'homme et un vecteur du genre Aedes. C. Liste des organes et fluides biologiques pour lesquels des particules virales ou de l'ARN viral ont été mis en évidence.

diaire d'Aedes aegypti. Mais Aedes albopictus (communément connu sous le nom de moustique tigre) est capable, expérimentalement, de répliquer le lignage africain. Une étude réalisée au Gabon a d'ailleurs montré la présence de ZIKV circulant chez ce moustique, présence corrélée à celle de virus dans le sérum de patients (Figure 3) [13, 14]. Toutefois, l'efficacité vectorielle d'Aedes albopictus pour le lignage asiatique, qui circule actuellement en Amérique du Sud et aux Caraïbes, reste à évaluer. Le virus se transmet aux arthropodes hématophages par repas sanguin. II est ensuite capable de se multiplier chez l'hôte vecteur sans l'affecter. II reste présent durant toute sa vie chez l'insecte, qui peut le transmettre aux animaux réservoirs au cours d'un nouveau repas sanguin. Une transmission transovarienne de certains flavivirus, notamment le DENV, a par ailleurs été démontrée chez des moustiques du genre Aedes [15]. II s'agit dans ce cas d'une transmission directe par la mère infectée à sa descendance.

\section{Les épidémies}

Différentes études sérologiques ont mis en évidence la circulation de ZIKV dans de nombreux pays d'Afrique et 
d'Asie [5]. Cependant, jusqu'en 2007, seuls quelques cas sporadiques ont été rapportés chez l'Homme. Le ZIKV n'a émergé que très récemment provoquant quatre épidémies: en Micronésie, sur l'île de Yap, en 2007 [1] ; en Polynésie française en 2013/2014 [16] ; en Nouvelle Calédonie en 2014 [17] ; et, enfin, l'épidémie actuelle qui a débuté au Brésil en 2015 [18] et qui s'étend depuis dans de nombreux pays de la région (Figure 4). Toutes ces épidémies récentes sont liées à des souches du lignage asiatique. Elles ont touché des populations immunologiquement naïves vis-à-vis de ce virus. L'épidémie qui a touché l'île de Yap a concerné 185 cas cliniquement évocateurs et confirmés par RT-PCR ou sérologie. L'enquête de séroprévalence a cependant estimé à plus de $73 \%$ la population infectée par ce virus [19] $]^{7}$. En Polynésie française, on estime à 32000 le nombre de patients ayant consulté pour une infection attribuée au ZIKV [16]. En Nouvelle Calédonie, 1400 cas ont été confirmés par RT-PCR lors de l'épidémie de 2014, mais une nouvelle épidémie, de moindre ampleur, est apparue en 2015 [17]. La proportion de patients asymptomatiques étant estimée à 70-80\%, l'ampleur de la circulation du virus est, de ce fait, probablement largement sous-estimée.

L'épidémie la plus récente qui se propage depuis 2015 a débuté dans le nord-est du Brésil. Plusieurs hypothèses ont été formulées quant à son origine. Elle pourrait résulter de contaminations lors de la coupe du monde de football qui a eu lieu en juin et juillet 2014, ou lors d'une compétition de course de pirogues polynésiennes ( $V a$ 'a) qui s'est tenue en août 2014 à laquelle plusieurs équipes du pacifique, dont la Polynésie française et la Nouvelle Calédonie, ont participé [20, 21]. Une analyse phylogénétique réalisée sur la souche circulante en Amérique latine montre qu'elle appartient également au lignage asiatique et qu'elle comporte 99,7\% d'homologie au niveau nucléotidique et $99,9 \%$ au niveau acides aminés, avec la souche qui circulait en Polynésie française en 2013-2014 [22], confirmant ainsi une relation étroite entre les deux épidémies. Le nombre de personnes touchées par l'infection ZIKV au Brésil (entre 2015 et 2016) représente entre 400000 et 1,5 millions d'individus selon les estimations. L'épidémie s'est par la suite étendue rapidement à de nombreuses zones : Barbade, Bolivie, Colombie, Équateur, Haïti, Salvador, Guadeloupe, Guatemala, Guyane, Honduras, Saint Martin, Martinique, Mexique, Panama, Paraguay, Porto Rico, Suriname, Vénézuela (Figure 4). Le second pays le plus touché par l'épidémie en Amérique latine, derrière le Brésil, est la Colombie avec 20000 cas répertoriés en quelques mois et une prévision par le ministère de la santé colombien de 600000 cas à terme.

Plusieurs cas importés d'infection ont été signalés en Amérique du Nord, en Australie et en Europe (Espagne, Royaume-Uni, Suisse, Italie, Pays-Bas, Danemark, Irlande) (Figure 4). En France métropolitaine, on dénombrait à la mi-février 2016, 18 cas dont 3 à Montpellier [23]. Tous ces malades ont été contaminés en Amérique du Sud ou aux Antilles et ne présentaient pas de complications particulières. L'extension de l'épidémie en Martinique, Guadeloupe et Guyane, fait craindre une augmentation importante du nombre de cas importés en métropole dans les prochains mois. Les risques d'introduction

${ }^{7}$ En 2010, l'île de Yap comptait 102600 habitants, soit près de 75000 individus contaminés. de ZIKV en métropole sont estimés réels comme le soulignait le haut conseil pour la santé publique dans un rapport publié en août 2015 [24], notamment en raison de la présence du moustique tigre, principalement dans le sud de la France. L'ampleur de l'épidémie actuelle ne fait qu'amplifier ce risque, d'autant plus que les Jeux Olympiques auront lieu en 2016 à Rio et qu'une récente simulation prédit la présence de virus circulant toute l'année dans certaines régions du Brésil [25]. Néanmoins, la probabilité de l'apparition d'une épidémie ZIKV en métropole est faible. Le plus probable est en effet l'apparition de foyers de cas autochtones isolés comme cela a été notamment le cas pour le CHIKV, à Montpellier en 2014. Ceci s'explique en particulier, par la plus faible densité de moustiques en France métropolitaine, même si le sud du pays reste le plus exposé, mais également par un programme de surveillance et de lutte anti-vectorielle et un vecteur sensible aux insecticides, ce qui n'est pas le cas pour Aedes aegypti présent dans les Antilles par exemple. En décembre 2015, l'ECDC (European Centre for Disease Prevention and Control), I'OMS (Organisation mondiale de la santé) et la PAHO (Pan American Health Organization) ont émis un bulletin d'alerte épidémiologique concernant l'épisode de Zika actuel et les désordres neurologiques qui sont associés à l'infection [11, 26]. En février 2016, l'OMS a décrété une urgence de santé publique de portée mondiale et a estimé le nombre de cas potentiel d'infection au ZIKV de 3 à 4 millions pour cette même année.

\section{Manifestations cliniques}

La période d'incubation après piqûre par un vecteur Aedes porteur du ZIKV est de 3 à 12 jours (d'après l'ECDC). Si la grande majorité des personnes infectées par ZIKV sont asymptomatiques (représentant 70-80\%), certaines vont développer une fièvre modérée (inférieure à $38,5^{\circ} \mathrm{C}$ ) et des symptômes de type pseudo-grippal relativement classiques pour les infections arbovirales, à savoir une éruption cutanée, des douleurs articulaires, des conjonctivites mais également des céphalées et des douleurs myalgiques. Ces symptômes relativement légers durent quelques jours et provoquent rarement une hospitalisation. Toutefois, plusieurs décès sont maintenant fortement suspectés d'être liés à l'infection par le ZIKV, notamment, plusieurs nouveau-nés (dont plus d'une centaine au Brésil) et au moins 5 adultes [11].

Au-delà de ces symptômes bien répertoriés, des symptômes neurologiques sévères seraient liés à l'infection par le ZIKV. Ainsi, des cas inhabituels de syndrome de 

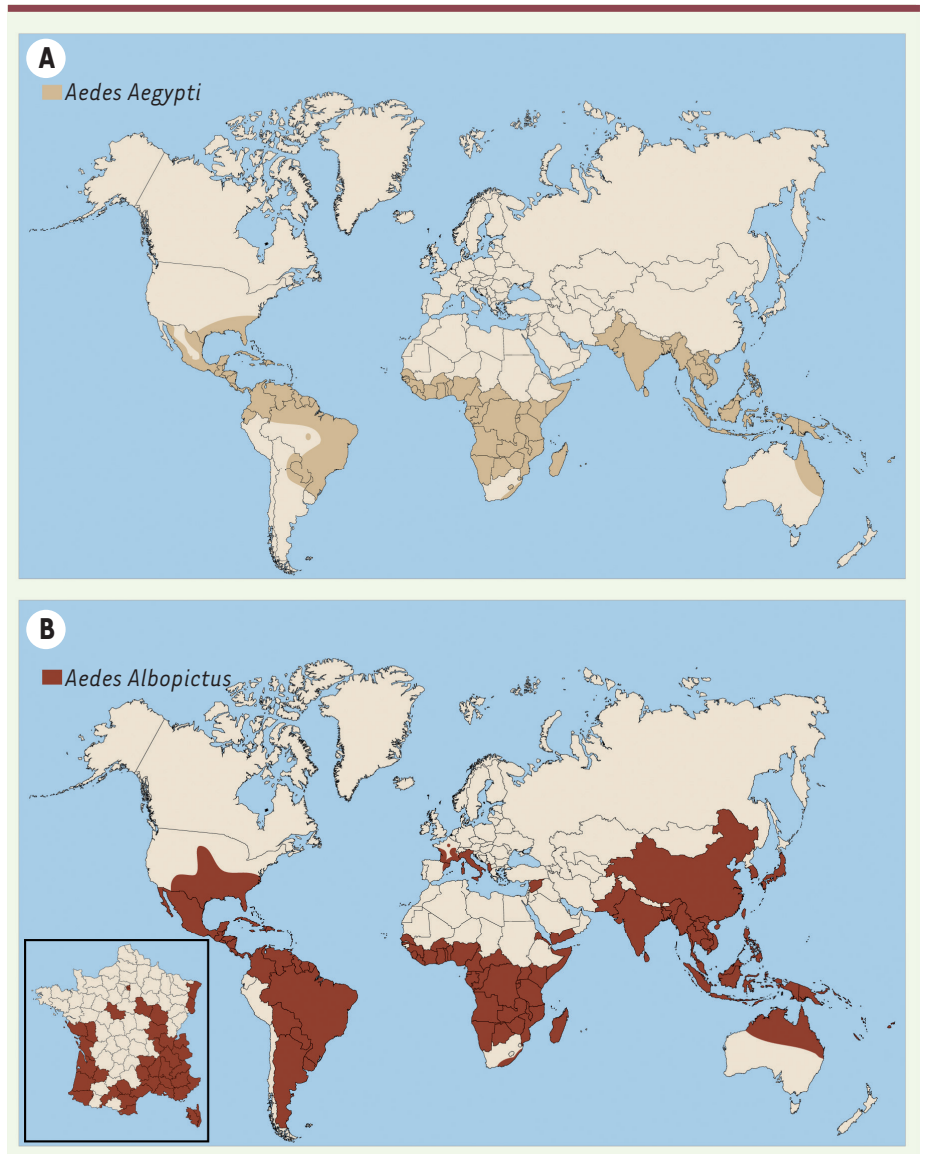

Figure 3. Répartition mondiale (A) d'Aedes aegypti et (B) d'Aedes albopictus.

Guillain-Barré8 ont été rapportés au moment de l'épidémie polynésienne [27]. Ils ont également été signalés chez certaines victimes brésiliennes, mais également au Salvador, en Colombie, au Vénézuela et en Martinique [28]. Le syndrome de Guillain-Barré est considéré comme un symptôme post-infectieux, dû à une réponse auto-immune de l'organisme et causant une paralysie neuromusculaire [29]. En particulier, il se manifeste par une faiblesse voire une paralysie progressive, pouvant atteindre les muscles respiratoires. Plusieurs décès liés directement au syndrome de Guillain-Barré chez des patients infectés par le ZIKV ont été rapportés en Colombie, soulignant ainsi la gravité des cas répertoriés. Le lien entre le ZIKV et le syndrome de GuillainBarré a été caractérisé par des études rétrospectives en Polynésie française [30]. II pourrait être, comme pour d'autres agents infectieux comme le WNV (West Nile virus) ou le cytomégalovirus, causé par une réponse immune aberrante qui entraînerait une détérioration des nerfs périphériques (par exemple par un processus de démyélination) [29].

\footnotetext{
${ }^{8}$ Le syndrome de Guillain-Barré est une atteinte des nerfs périphériques caractérisée par une faiblesse, voire une paralysie progressive, débutant le plus souvent au niveau des jambes et remontant parfois jusqu'à atteindre les muscles de la respiration puis les nerfs de la tête et du cou. Ce syndrome est aussi appelé polyradiculonévrite aiguë inflammatoire, ou encore polyradiculonévrite aiguë post-infectieuse car il survient souvent après une infection. Dans la majorité des cas, les personnes atteintes récupèrent leurs capacités physiques au bout de 6 à 12 mois.
}

Un autre symptôme neurologique particulièrement inquiétant est la description de cas de microcéphalies chez des nouveau-nés. Ce symptôme est diagnostiqué lorsque la circonférence de la tête du fœtus est inférieure de 2 à 3 écarts-types par rapport à la normale. Début février 2016, plus de 4000 cas potentiels probablement liés à une infection ayant eu lieu au cours du premier ou du deuxième trimestre de grossesse, ont été recensés au Brésil [31] contre une centaine de cas en 2014. La microcéphalie est une affection congénitale pouvant être d'origine génétique ou sporadique comme à la suite d'une infection par le cytomégalovirus ou d'une toxoplasmose [32]. Si aucun lien direct entre ZIKV et microcéphalie n'avait été clairement démontré début 2016, des bébés microcéphales, nés de mères symptomatiques durant leur grossesse, ont été diagnostiqués séropositifs au virus [33]. La présence de virus dans le cerveau d'un bébé microcéphale mort-né permet d'envisager qu'une infection intra-utérine serait à l'origine de ces malformations congénitales [34-36]. Des lésions rétiniennes associées à certains cas de microcéphalie suggèrent par ailleurs, une atteinte directe par le virus [37, 38]. Des études en cours sur 35 bébés microcéphales devraient apporter très prochainement plus d'informations [39]. Enfin, des études rétrospectives portant sur l'épidémie polynésienne ont mis en évidence une dizaine de cas de microcéphalie et de malformations du tronc cérébral [40]. Dans ce contexte, les agences de santé publique de différents pays comme le CDC (Center for Disease Control) aux États-Unis, ont émis des alertes conseillant aux femmes enceintes de ne pas se rendre dans les pays où le virus est présent, ou même, de reporter la date de conception, soulignant ainsi l'importance majeure de cette problématique.

\section{Transmission interhumaine}

Si la virémie (c'est-à-dire la présence dans le sang) du virus Zika est relativement courte (entre 3 et 5 jours), des virions ont cependant été détectés dans plusieurs autres liquides biologiques incluant les urines (10 jours post-infection) [41], la salive (3 à 5 jours) [42] et le sperme (15 jours post-infection) [43] (Figure 2). La possibilité de transmission du virus par transfusion sanguine a été présentée comme un facteur de risque et des études permettant de déterminer la réelle prévalence du virus chez les donneurs de sang dans les zones endémiques et de leur capacité à transmettre le virus à des receveurs sont attendues [44]. Les premières analyses effectuées durant l'épidémie en Polynésie ont montré que $3 \%$ des donneurs de sang, 


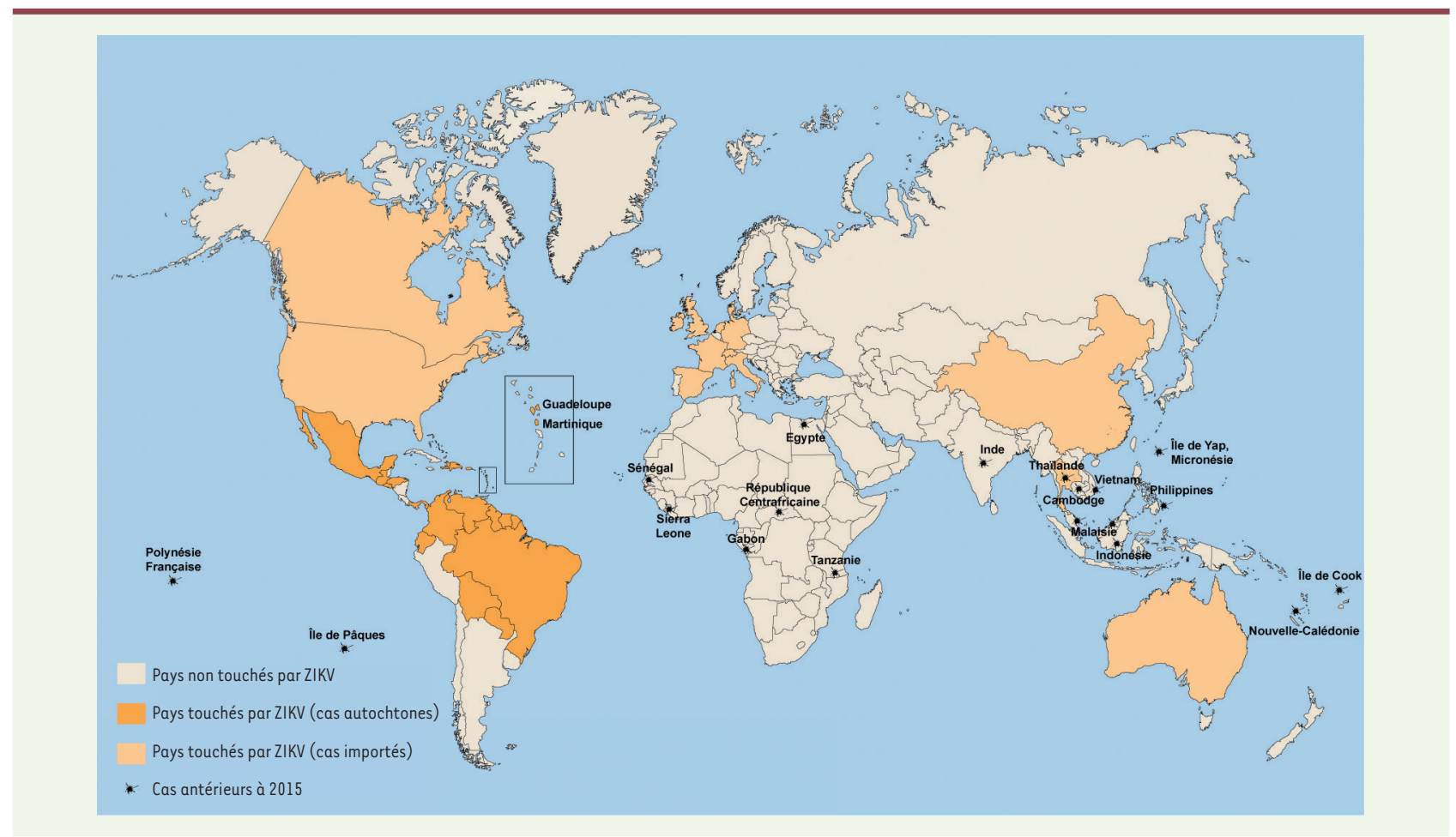

Figure 4. Répartition mondiale des pays touchés par le virus Zika (ZIKV). Sont représentés en orange foncé les cas autochtones et en orange clair les cas importés lors de l'épidémie 2015/2016. Les cas antérieurs sont également identifiés.

asymptomatiques, étaient détectés positifs au ZIKV [45]. L'efficacité des systèmes de surveillance conduit aujourd'hui à la mise en place de mesures préventives avec notamment la mise en place de dépistage moléculaire du virus chez les donneurs de sang dans les zones endémiques. Ainsi, tous les dons prélevés aux Antilles et en Guyane font l'objet d'un dépistage du ZIKV réalisé en métropole par un laboratoire référent.

Les transmissions périnatales et notamment placentaires sont également fortement suspectées. Plusieurs enfants ont ainsi probablement été contaminés au cours de la délivrance ou par transmission placentaire, comme cela a été rapporté dans une étude portant sur l'épidémie polynésienne [46]. Deux mères ont présenté des symptômes de l'infection soit peu avant, soit peu après l'accouchement, ainsi que I'un des deux bébés, 3 jours après l'accouchement. La présence d'ARN viral Zika a été confirmée dans le sang des deux mères mais aussi des enfants, suggérant fortement une transmission périnatale [46]. Du virus réplicatif a également été mis en évidence dans du lait maternel d'une femme infectée en Nouvelle Calédonie [47]. Ces modes de transmission (périnatal et/ou par allaitement maternel) ont aussi été rapportés pour d'autres arbovirus tels que le CHIKV [48], le DENV [49] ou le WNV [50].

Plusieurs cas rapportés suggèrent une possible transmission sexuelle du ZIKV, une voie de transmission jamais décrite à ce jour pour un arbovirus. La découverte du virus dans le sperme d'un patient polynésien de 44 ans, 15 jours après le début des symptômes, fait suspecter une transmission par voie sexuelle [43]. Deux cas importés américains confortent notamment cette hypothèse : celui d'un patient ayant contracté le virus au Sénégal et l'ayant transmis à son retour aux États-Unis à sa femme pourtant restée sur place (la transmission vectorielle étant ainsi écartée) [51] et celui d'un patient texan infecté au Venezuela ayant transmis le virus à sa partenaire qui n’avait pas, non plus, séjourné dans une zone épidémique. En France métropolitaine, un cas de transmission sexuelle du ZIKV a également été rapporté.

\section{Diagnostic}

Le diagnostic d'une infection à ZIKV présente certaines difficultés. La première est inhérente aux nombreuses caractéristiques cliniques et épidémiologiques que partage le ZIKV avec d'autres arboviroses. En effet, lorsqu'ils sont présents, les signes cliniques se rapprochent de ceux observés pour de nombreuses autres fièvres arbovirales. Ces virus partageant les mêmes vecteurs Aedes, leurs zones géographiques d'endémies sont donc généralement superposables (Figure 3). Ainsi les arguments cliniques et épidémiologiques du diagnostic ne sont que des éléments d'orientation vers une fièvre qui est liée à une infection par arbovirus. Ils devront donc être confirmés par des tests permettant le ciblage du virus lui-même, mais aussi explorant la 
réponse immune de l'hôte. Le développement de tests nucléiques de type multiplexe (révélant plusieurs paramètres dans un même échantillon), discriminant une infection ayant pour origine le ZIKV des autres infections par des arboviroses, sera donc de première importance. À ce jour, la nécessité d'établir un diagnostic étiologique différentiel entre diverses arboviroses était, au-delà de l'intérêt épidémiologique, justifiée essentiellement par la potentielle gravité de l'infection par le virus de la dengue qui nécessitait une prise en charge singulière. Les récentes données évoquant un lien entre l'infection par ZIKV et des pathologies neurologiques de type Guillain-Barré, mais surtout la probable tératogénicité de ce virus, révélée par l'épidémie actuelle en Amérique du Sud, ne font que renforcer les besoins en un diagnostic de laboratoire fiable.

Comme pour la plupart des infections causées par les autres arboviroses, le diagnostic biologique de l'infection mobilise des techniques virologiques directes, lorsque le patient est vu dans les premiers jours qui suivent l'installation des signes cliniques (généralement jusqu'à 5-7 jours), et, au-delà de ces 5 à 7 jours, des techniques sérologiques qui permettent le plus souvent de rattraper un diagnostic plus tardif (Figure 5). En ce qui concerne les tests virologiques, bien que le ZIKV se réplique aisément dans des modèles cellulaires simples (comme les cellules de primate Vero ${ }^{9}$ ou les cellules d'insectes $\mathrm{C} 6 / 36$, un clone issu d'Aedes albopictus), la culture n'est pas utilisée en diagnostic de routine. Désormais, ce diagnostic repose sur des analyses moléculaires (par RT-PCR). Cependant, à ce jour, il n'y a pas de techniques consensuelles. Les laboratoires développent en effet de nombreuses approches «maison», la plus représentée étant celle mise au point par Lanciotti et al. lors de l'épidémie qui a touché la Micronésie et qui cible uniquement la souche asiatique de ZIKV [1]. Des solutions commerciales, validées, commencent néanmoins à apparaître sur le marché. La détection d'une virémie révélée par RT-PCR pose le diagnostic étiologique. Ces tests peuvent en effet être facilement pris en défaut, la virémie de ZIKV est fugace et semble détectable uniquement dans les 3 à 5 jours qui suivent le début des signes cliniques [42]. De nombreuses études ont montré que la détection du virus dans les urines pouvait être toutefois particulièrement pertinente. En effet, l'excrétion du virus par les voies urinaires peut être mise en évidence jusqu'à 10 jours après le début des signes cliniques (Figure 5) [41].

L'absence de détection du virus n'exclut pas l'infection virale, surtout si les prélèvements analysés ont été réalisés tardivement par rapport à l'apparition des signes cliniques. À ce jour, les données sur les cinétiques d'apparition des anticorps anti-ZIKV (IgM et $I g G)$ produits à la suite de l'infection sont encore parcellaires. Néanmoins, le recours à la sérologie reste d'intérêt, notamment dans certaines indications particulières. II n'y a pas de techniques commerciales validées pour le diagnostic sérologique à ce jour, même si certaines trousses ELISA ou solutions par immunofluorescence commencent à être proposées. Actuellement, les sérologies sont réalisées principalement par des centres spécialisés. Elles font appel à des techniques d'immunofluorescence indirecte utilisant

${ }^{9}$ Lignée cellulaire de rein de singe vert d'Afrique. des cultures de cellules infectées par le ZIKV [52] ou des techniques «maison » d'ELISA par immunocapture (utilisant des anticorps spécifiques fixant et révélant des antigènes viraux), là encore réalisées à partir de stocks de virus produits dans certains laboratoires référents. La principale limite des approches sérologiques est la survenue de nombreuses réactions croisées qui peuvent interférer avec les autres flavivirus en raison de leur importante communauté antigénique [53]. L'interprétation des sérologies est donc souvent délicate, en particulier dans les zones d'endémicité des autres flavirirus. Ces réactions croisées peuvent également être observées chez des individus ayant reçu une vaccination amarile (vaccination contre le virus de la fièvre jaune). En fait, à ce jour, seule l'utilisation d'approches de séroneutralisation virale permet de conclure quant à la spécificité antiZIKV des anticorps détectés [23]. Ces techniques, qui sont extrêmement lourdes, ne sont que difficilement accessibles à la plupart des laboratoires. Ces réserves sur les approches sérologiques sont donc un obstacle majeur dans l'établissement d'un diagnostic rétrospectif. Elles compliquent significativement un éventuel dépistage. Cela pose des problèmes, notamment pour confirmer l'étiologie, post-infection par le Zika, de certains syndromes neurologiques qui surviennent dans de rares cas, à distance de la primo-infection et qui sont souvent asymptomatiques ou cliniquement discrets. Une autre difficulté qui pourrait devenir très préoccupante concerne la possibilité de mettre en place une éventuelle stratégie de dépistage chez des femmes enceintes qui auraient pu être exposées au ZIKV au cours de leur grossesse [54].

\section{Perspectives}

Les défis scientifiques, notamment dans la compréhension de la pathogenèse liée au ZIKV, sont immenses. L'un des premiers sera de confirmer, expérimentalement, le lien existant entre le ZIKV et les cas de désordres neurologiques qui y sont associés. Même si l'étiologie virale est fortement suspectée, les évidences scientifiques ne sont pas suffisamment robustes pour confirmer la relation entre virus et atteintes neurologiques. Une confirmation de cette relation nécessitera probablement le développement de modèles animaux. II n'existe pas, à I'heure actuelle, de traitement antiviral ou de vaccin contre le ZIKV. La prophylaxie repose donc uniquement sur une thérapeutique symptomatique, notamment par l'utilisation d'antalgiques. Plusieurs groupes pharmaceutiques dont Sanofi Pasteur ont déjà annoncé 


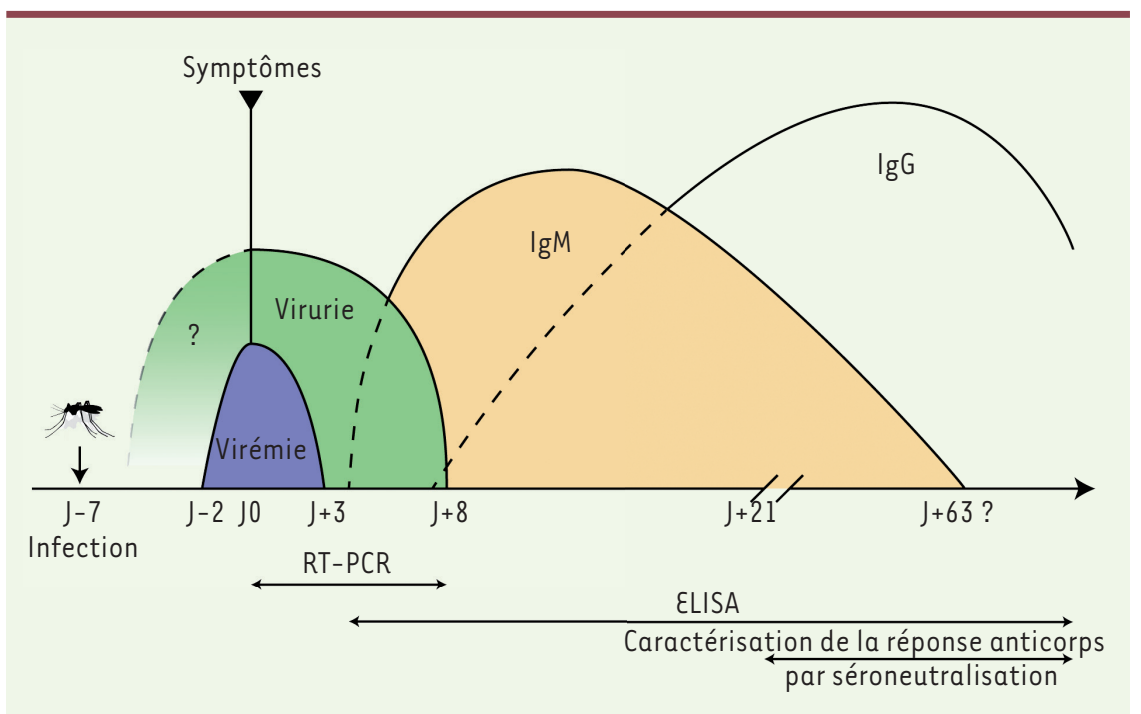

Figure 5. Cinétique de marqueurs biologiques (virologiques et sérologiques) : support diagnostique (adapté du Centre National de Référence) $\lg (M$ ou $G)$ : immunoglobulines $(M$ ou $G) ; J \pm n$ : jour par rapport à l'apparition des symptômes; RT-PCR : reverse transcriptase-polymerase chain reaction.

viable. Cette approche, qui est efficace localement, doit cependant faire ses preuves à grande échelle.

L'effort planétaire entrepris pour surveiller, prévenir et comprendre l'infection ZIKV est justifié par l'émergence de cette menace virale. Elle signe, aujourd'hui, les risques d'une nouvelle pandémie. $\diamond$

leur intention de développer un vaccin contre le ZIKV, ce groupe pharmaceutique français comptant profiter de l'expertise acquise pour l'élaboration d'un vaccin anti-dengue (Dengvaxia ${ }^{\circledR}$ ) qui a été autorisé sur le marché brésilien à la fin de l'année 2015. Le groupe GSK (GlaxoSmithKline) a également annoncé son intention d'étudier la faisabilité d'un vaccin anti-ZIKV. L'institut américain des allergies et maladies infectieuses (NIAID) travaille par ailleurs, sur deux approches différentes (un vaccin à ADN et un vaccin vivant atténué). Cependant, pour la dengue, le développement d'un vaccin a dû faire face à deux obstacles majeurs : la variabilité du virus DENV (il compte en effet 5 sérotypes) et l'existence d'anticorps facilitants, qui, présents - à la suite d'une immunité vaccinale ou naturelle - peuvent en fait aggraver l'infection, en particulier en induisant des formes hémorragiques par un mécanisme d'ADE (antibodydependent enhancement) favorisant la pénétration du virus par les anticorps ainsi produits. Bien que le phénomène d'ADE n'ait jamais été démontré dans l'infection par le ZIKV, il est probable que ce type de mécanisme influera sur le développement d'un vaccin, sûr et efficace, contre le ZIKV. L'élaboration d'un vaccin devrait de toute façon prendre plusieurs années pour son développement. Comme pour de nombreuses autres arboviroses, le caractère soudain et imprévisible des épidémies à ZIKV rendent une approche vaccinale prophylactique prohibitive en terme de coût. À ce jour, la lutte antivectorielle reste ainsi le moyen le plus efficace pour combattre ces virus. L'utilisation massive d'insecticides qui sont essentiellement à visée adulticide, a fait ses preuves dans le contrôle des infections, notamment pour le virus de la fièvre jaune. Cependant, cette stratégie est limitée par des problèmes de résistance des vecteurs mais également par leur «urbanisation », conduisant à l'impossibilité de leur éradication dans des zones qui sont très densément peuplées. Dans ces zones, la sensibilisation à la réduction des gîtes larvaires et les mesures de protection individuelle resteront vraisemblablement les plus efficaces. Une stratégie consisterait à effectuer des lâchers massifs de vecteurs Aedes aegypti mâles qui auraient été modifiés transgéniquement afin d'engendrer une progéniture non-

\section{SUMMARY}

Zika virus, an emerging threat

Zika virus, discovered in 1947, is particularly publicized because of its involvement in a major epidemic that began in 2015 and which epicenter is located in Latin America, mainly in Brazil. In the majority of cases (70$80 \%$ ) the infection is asymptomatic, however in some patients, moderate fever, skin rash, conjunctivitis and myalgia may occur. More alarming, neurological complications are reported, in particular cases of microcephaly probably resulting from the infection of women in the first or second trimester of pregnancy. Moreover, Guillain-Barré syndromes have also been identified in patients whose infection was confirmed. The extent of the current outbreak reveals the very primitive state of knowledge about the pathophysiology of this virus. Thus, a global effort is being undertaken in order to quickly characterize the molecular interaction of the virus with human cells, but also to develop specific diagnostic assays and vaccinal approaches. $\diamond$

\section{LIENS D'INTÉRÊT}

Les auteurs déclarent n'avoir aucun lien d'intérêt concernant les données publiées dans cet article.

\section{RÉFÉRENCES}

1. Lanciotti RS, Kosoy OL, Laven JJ, et al. Genetic and serologic properties of Zika virus associated with an epidemic, Yap State, Micronesia, 2007. Emerg Infect Dis 2008; $14: 1232-9$.

2. Chambers TJ, Hahn CS, Galler R, Rice CM. Flavivirus genome organization, expression, and replication. Annu Rev Microbiol 1990 ; 44 : 649-88.

3. Dick GWA, Kitchen SF, Haddow AJ. Zika virus. I. Isolations and serological specificity. Trans R Soc Trop Med Hyg $1952 ; 46: 509-20$.

4. Macnamara FN. Zika virus: a report on three cases of human infection during an epidemic of jaundice in Nigeria. Trans R Soc Trop Med Hyg 1954 ; $48: 139-45$. 


\section{RÉFÉRENCES}

5. Faye 0 , Freire CCM, Iamarino A, et al. Molecular evolution of Zika virus during its emergence in the $20^{\text {th }}$ century. PLoS Negl Trop Dis $2014 ; 8:$ e2636.

6. Baronti C, Piorkowski G, Charrel RN, et al. Complete coding sequence of zika virus from a French polynesia outbreak in 2013. Genome Announc 2014 ; 2 : piie00500-14.

7. Buckley A, Gould EA. Detection of virus-specific antigen in the nuclei or nucleoli of cells infected with Zika or Langat virus. J Gen Virol $1988 ; 69: 1913-20$.

8. Hamel R, Dejarnac 0 , Wichit $\mathrm{S}$, et al. Biology of Zika virus infection in human skin cells. J Virol $2015 ; 89: 8880-96$.

9. Dick GWA. Zika virus. II. Pathogenicity and physical properties. Trans R Soc Trop Med Hyg 1952 ; $46: 521-34$.

10. Bell TM, Field $\varepsilon$, Narang HK. Zika virus infection of the central nervous system of mice. Arch Gesamte Virusforsch $1971 ; 35: 183-93$.

11. World Health Organization. Neurological syndrome, congenital malformations, and Zika virus infection. Implications for public health in the Americas. In : Epidemiological Alert. Geneva : WHO, 2015. http://www.paho.org/hq/index.php?option=com_docman\&task=doc_view\&ltemid=270\&gid =32405\&lang=en

12. Darwish MA, Hoogstraal H, Roberts TJ, et al. A sero-epidemiological survey for certain arboviruses (Togaviridae) in Pakistan. Trans R Soc Trop Med Hyg 1983 ; 77 : 442-5.

13. Wong PSJ, Li MZI, Chong CS, et al. Aedes (Stegomyia) albopictus (Skuse): a potential vector of Zika virus in Singapore. PLoS Negl Trop Dis $2013 ; 7: 1-5$.

14. Grard G, Caron M, Mombo IM, et al. Zika virus in Gabon (Central Africa)-2007: a new threat from Aedes albopictus? PLoS Negl Trop Dis $2014 ; 8$ : 1-6.

15. Rosen L, Shroyer DA, Tesh RB, et al. Transovarial transmission of dengue viruses by mosquitoes: Aedes albopictus and Aedes aegypti. Am J Trop Med Hyg 1983 ; 32 : 1108-19.

16. Mallet HP, Vial AL, Musso D. Bilan de l'épidémie à virus Zika en Polynésie française, 2013-2014. Bull Inf Sanit Epidemiol Stat $2015 ; 13: 1-5$.

17. Roth $A$, Mercier A, Lepers $C$, et al. Concurrent outbreaks of dengue, chikungunya and Zika virus infections: an unprecedented epidemic wave of mosquito-borne viruses in the Pacific 2012-2014. Euro Surveill 2012 ; 19 : pii20929.

18. Zanluca C, de Melo VCA, Mosimann ALP, et al. First report of autochthonous transmission of Zika virus in Brazil. Mem Inst Oswaldo Cruz 2015 ; 110 : 569-72.

19. Duffy MR, Chen TH, Hancock WT, et al. Zika virus outbreak on Yap Island, Federated States of Micronesia. N Engl J Med 2009 ; 360 : 2536-43.

20. Marcondes CB, Ximenes MF. Zika virus in Brazil and the danger of infestation by Aedes (Stegomyia) mosquitoes. Rev Soc Bras Med Trop 2015 ; piiS0037-86822015005003102.

21. Musso D. Zika virus transmission from French Polynesia to Brazil. Emerg Infect Dis 2015 ; 21 : 1887.

22. Enfissi A, Codrington J, Roosblad J, et al. Zika virus genome from the Americas. Lancet 2016 ; 387 227-8

23. Maria AT, Maquart M, Makinson A, et al. Zika virus infections in three travellers returning from South America and the Caribbean respectively, to Montpellier, France, December 2015 to January 2016. Euro Surveill 2016 ; 2 : pii30131.

24. Haut Conseil de la Santé Publique. Prise en charge médicale des personnes atteintes par le virus Zika. Paris : Haut Conseil de la Santé Publique, 1995. http://www.hcsp.fr/explore.cgi/ avisrapportsdomaine? clefr $=517$

25. Bogoch II, Brady 0J, Kraemer MUG, et al. Anticipating the international spread of Zika virus from Brazil. Lancet 2016 ; 387: 335-6.

26. $\varepsilon C D C$. Zika virus epidemic in the Americas: potential association with microcephaly and Guillain Barré syndrome. In : Rapid risk assessment. ECDC, 2015. Available: http://ecdc.europa.eu/en/ publications/Publications/zika-virus-americas-association-with-microcephaly-rapid-riskassessment.pdf

27. Oehler $\varepsilon$, Watrin L, Larre $P$, et al. Zika virus infection complicated by Guillain-Barre syndrome-case report, French Polynesia, December 2013. Euro Surveill $2014 ; 19$ : pii20720.

28. ECDC. Zika virus disease epidemic: potential association with microcephaly and Guillain-Barré syndrome (second update). Rapid risk assessment. ECDC, 2016. Available at: http://ecdc.europa eu/en/publications/Publications/zika-virus-rapid-risk-assessment-8-february-2016.pdf

29. Van Doorn PA, Ruts L, Jacobs BC. Clinical features, pathogenesis, and treatment of Guillain-Barré syndrome. Lancet Neurol $2008 ; 7: 939-50$.

30. Cao-Lormeau VM, Blake A, Mons S, et al. Guillain-Barré syndrome outbreak associated with Zika virus infection in French Polynesia: a case-control study. Lancet 2016 ; pii: S0140-6736(16)00562-6.

31. COES-microcefalias. Monitoramento dos casos de microcefalia no Brasil. Informe epidemiologico $n^{\circ} 10$ - Semana epidemiologica (SE) 03/2016 (17 A 23/01/2016) 2016. Available at: http://www. salud.gob.ec/wp-content/uploads/2015/12/COES-Microcefalias-Informe-Epidemiol-gico-10-SE03-2016-27jan2016-12h04.pdf

32. Adams Waldorf KM, McAdams RM. Influence of infection during pregnancy on fetal development. Reproduction 2013 ; 146 : R151-62.

33. Oliveira Melo AS, Malinger G, Ximenes R, et al. Zika virus intrauterine infection causes fetal brain abnormality and microcephaly: tip of the iceberg ? Ultrasound Obstet Gynecol 2016 ; 47 : 6-7.

34. Mlakar J, Korva M, Tul N, et al. Zika Virus associated with microcephaly. $N$ Engl J Med 2016 ; 374 : 951-8.
35. Brasil Martines R, Bhatnagar J, Keating MK, et al. Notes from the field: Evidence of Zika virus infection in brain and placental tissues from two congenitally infected newborns and two fetal losses - Brazil, 2015. MMWR Morb Mortal Wkly Rep $2016 ; 65: 1-2$.

36. $\varepsilon C D C$. Zika virus disease epidemic: potential association with microcephaly and Guillain-Barré syndrome (first update). Rapid risk assessment. ECDC, 2016. Available at: http://ecdc.europa.eu/en/publications/Publications/ rapid-risk-assessment-zika-virus-first-update-jan-2016.pdf

37. Ventura CV, Maia M, Bravo-Filho V, et al. Zika virus in Brazil and macular atrophy in a child with microcephaly. Lancet $2016 ; 387: 228$

38. Ventura CV, Maia M, Ventura BV, et al. Ophthalmological findings in infants with microcephaly and presumable intra-uterus Zika virus infection. Arq Bras Oftalmol $2016 ; 79$ : 1-3.

39. Schuler-Faccini L, Ribeiro EM, Feitosa IML, et al. Possible Association Between Zika Virus Infection and Microcephaly-Brazil, 2015. MMWR Morb Mortal Wkly Rep 2016 ; 65 : 59-62.

40. BVS, CHPF. Note sur les investigations autour des malformations cérébrales congénitales ayant suivi l'épidémie de zika de 2013-2014. 2015. Available at: http://www.hygiene-publique.gov.pf/IMG/pdf/note_malformations_ congenitales_cerebrales.pdf

41. De M. Campos R, Cirne-Santos C, Meira GLS, et al. Prolonged detection of Zika virus RNA in urine samples during the ongoing Zika virus epidemic in Brazil. J Clin Virol 2016; 77 : 69-70.

42. Musso D, Roche $C$, Nhan TX, et al. Detection of Zika virus in saliva. J Clin Virol $2015 ; 68: 53-5$

43. Musso D, Roche $C$, Robin $\varepsilon$, et al. Potential sexual transmission of Zika virus. Emerg Infect Dis $2015 ; 21: 359-61$.

44. Marano G, Pupella S, Vaglio S, et al. Zika virus and the never-ending story of emerging pathogens and transfusion medicine. Blood Transfus 2016; 14 95- 100 .

45. Musso D, Nhan T, Robin $\varepsilon$, et al. Potential for Zika virus transmission through blood transfusion demonstrated during an outbreak in French Polynesia, November 2013 to February 2014. Euro Surveill $2014 ; 19$ : pii20761.

46. Besnard $M$, Lastère $S$, Teissier $A$, et al. Evidence of perinatal transmission of zika virus, French Polynesia, December 2013 and February 2014. Euro Surveill $2014 ; 19$ : pii20761.

47. Dupont-Rouzeyrol M, Biron A, O'Connor 0 , et al. Infectious Zika vira particles in breastmilk. Lancet 2016; piiS0140-6736(16)00624-3.

48. Gérardin P, Barau G, Michault A, et al. Multidisciplinary prospective study of mother-to-child chikungunya virus infections on the island of La Réunion. PLoS Med 2008 ; 5 : e60.

49. Tan PC, Rajasingam G, Devi S, Omar SZ. Dengue infection in pregnancy: prevalence, vertical transmission, and pregnancy outcome. Obstet Gynecol $2008 ; 111: 1111-7$.

50. Hayes $\varepsilon B$, Komar N, Nasci RS, et al. Epidemiology and transmission dynamics of West Nile virus disease. Emerg Infect Dis 2005 ; 11 : 1167-73.

51. Foy BD, Kobylinski KC, Foy JLC, et al. Probable non-vector-borne transmission of Zika virus, Colorado, USA. Emerg Infect Dis 2011 ; 17 : 880-2.

52. Zammarchi L, Tappe D, Fortuna C, et al. Zika virus infection in a traveller returning to Europe from Brazil, March 2015. Euro Surveill $2015 ; 20$ : pii21153.

53. Gould EA, Solomon T. Pathogenic flaviviruses. Lancet 2008 ; $371: 500-9$.

54. Petersen $દ \varepsilon$, Staples JE, Meaney-Delman D, et al. Interim guidelines for pregnant women during a Zika virus outbreak - United States, 2016. MMWR Morb Mortal Wkly Rep $2016 ; 65$ : 30-3.

55. Flamand M, Desprès $P$. La dengue. Med Sci (Paris) 2002 ; 18 : 816-8.

56. Consigny PH, Lecuit $M$, Lortholary 0 . Infection par le virus Chikungunya : une alphavirose ré-émergente. Med Sci (Paris) 2006 ; 22 : 444-6.

57. Lanteri MC, Assal A, Norris PJ, Busch MP. Le virus West Nile. I. La conquête de l'Ouest. Med Sci (Paris) $2011 ; 27: 375-81$
TIRÉS À PART

y. Simonin 